\title{
Observing Oceans in Tightly Packed Planetary Systems: Perspectives from Polarization Modeling of the TRAPPIST-1 System
}

\author{
Pushkar Kopparla ${ }^{1}$ (D), Vijay Natraj ${ }^{2}$ (D), David Crisp ${ }^{2}$ (D), Kimberly Bott ${ }^{3,4}$, Mark R Swain ${ }^{2}$, and Yuk L Yung ${ }^{1,2}$ \\ ${ }^{1}$ Division of Geological and Planetary Sciences, Caltech, Pasadena, CA, USA; pushkar@caltech.edu \\ 2 Jet Propulsion Laboratory, California Institute of Technology, Pasadena, CA, USA \\ ${ }^{3}$ Virtual Planetary Laboratory, Seattle, WA, USA \\ ${ }^{4}$ University of Washington, Seattle, WA, USA \\ Received 2018 February 21; revised 2018 August 8; accepted 2018 August 8; published 2018 September 10
}

\begin{abstract}
The recently discovered TRAPPIST-1 system is exciting due to the possibility of several rocky, Earth-sized planets harboring liquid water on their surface. To assess the detectability of oceans on these planets, we model the diskintegrated phase curves and polarization signals for planets in this system for reflected starlight. We examine four cases: (1) dry planet, (2) cloud-covered planet, (3) planet with regional-scale oceans, and (4) planet with global oceans. Polarization signals are strongest for optically thin $(\lesssim 0.1)$ atmospheres over widespread oceans, with the degree of polarization being up to $90 \%$ for a single planet or on the order of 100 parts per billion for the star-planet system. In cases where reflected light from different planets in a tightly packed system cannot be separated, observing in polarized light allows for up to a tenfold increase in star-planet contrast compared to photometric observations alone. However, polarization from other sources, such as atmospheric scattering and cloud variability, will pose major challenges to the detection of glint (specularly reflected starlight) polarization signals. Planned telescopes like LUVOIR may be capable of observing glint from Earth-like planets around Sun-like stars, and if equipped with a polarimeter can significantly improve our ability to detect and study oceans on rocky exoplanets.
\end{abstract}

Key words: planetary systems - planets and satellites: oceans - polarization

\section{Introduction}

The discovery of the TRAPPIST-1 system, with several rocky planets tightly packed in a resonant chain of orbits around an ultracool dwarf star (effective temperature of about $2550 \mathrm{~K}$ ), is the first of its kind (Gillon et al. 2017; Luger et al. 2017). The close-in orbits of these planets combined with the cool temperature of the star allow several of these planets to receive stellar insolation levels comparable to the solar flux at Earth. This circumstance has inspired several studies on different aspects of habitability for these planets such as atmospheric greenhouse effects (Meadows et al. 2017; Wolf 2017), surface UV fluxes (O'Malley-James \& Kaltenegger 2017) and even some studies on the effects of vegetation (Alberti et al. 2017) and possible exchange of biological matter between planets in the system (Lingam \& Loeb 2017).

In this work, we focus on finding directly detectable signals for liquid water (such as specularly reflected starlight from the surface, commonly known as glint) for planets in this system. Looking for glint in reflected starlight was first proposed by Cowan et al. (2009), Robinson et al. (2010). However, it is possible to have false positives mimicking glint from a liquid ocean surface occurring due to reflection off ice or snow at high latitudes (Cowan et al. 2012). In such cases, an additional piece of information from polarization can help resolve the degeneracy. Stam (2008) was the first to propose a search for glint in polarized light. Later work explored the parameter space of various atmospheric and surface conditions in the context of detectability, and concluded that the near-IR atmospheric windows from $1.55-1.75 \mu \mathrm{m}$ and $2.1-2.3 \mu \mathrm{m}$ were the most favorable to detect glint reflection from the surface for an Earth-like atmosphere with clouds and aerosols (Zugger et al. 2010, 2011).
Recent work on modeling the climates of the TRAPPIST-1 planets found that planet $\mathrm{d}$ was most likely to have liquid water, with the outer planets being more likely to be frozen over (Alberti et al. 2017). However, other work on estimating the water loss rates on the planets of this system indicate that planets e to $\mathrm{h}$ can hold onto some of their water, while the inner planets have loss rates that are too high to expect oceans to exist (Bourrier et al. 2017). We therefore focus much of our modeling on TRAPPIST-1e, as a likely candidate for possessing some liquid water on the basis of these studies.

In the following section, we describe our vector radiative transfer model. Then we present polarized phase curves for a few cases of an ocean on the surface and the magnitude of an observable signal for various atmospheric conditions. Following that, we explore the issues associated with isolating the glint signal from a single planet in a tightly packed multiplanet system. We discuss our findings and implications for future observations in the final section.

\section{Model}

A detailed description of the polarized radiative transfer model and its validation can be found in an earlier paper (Kopparla et al. 2016), but we summarize it here for convenience. The core of the model is the one-dimensional (1D), multiple scattering, vector radiative transfer model, VLIDORT (Spurr 2006). The planet's surface is divided up into a grid of boxes (typically 64 boxes) based on a quadrature scheme (Horak 1950), and the radiation field is derived at each box using a plane parallel one-dimensional atmosphere. The radiation fields from each box are then summed over the entire surface to give the disk-integrated intensity and polarization values. To simulate ocean-covered surfaces, we have used the Cox-Munk ocean reflection glint surface model (Cox \& 

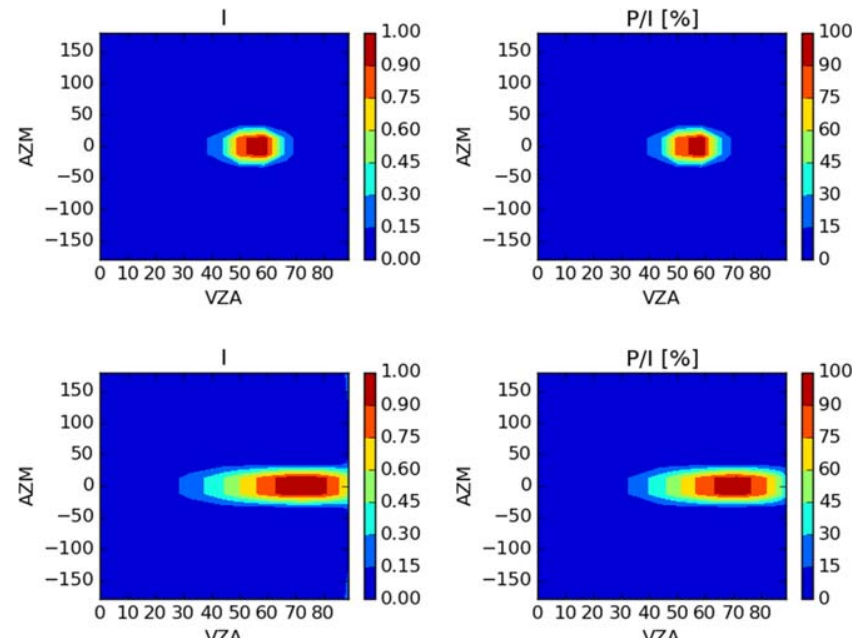

Figure 1. Normalized Stokes parameter $I$ and degree of polarization $P$ for a Cox-Munk glint surface under a very thin 1D Rayleigh scattering atmosphere (optical depth 0.001) for a variety of values of VZA and AZM. SZA is held fixed at $60^{\circ}$. The wind speed is $1 \mathrm{~m} \mathrm{~s}^{-1}$ for the plots in the top row, and $10 \mathrm{~m} \mathrm{~s}^{-1}$ for those in the bottom row. Mean wind speeds over oceans on Earth lie between these values (Atlas et al. 2011).

Munk 1954) capability in VLIDORT. The model outputs the Stokes parameters $I$ (intensity), $Q$ and $U$ (linear polarization parameters), and $V$ (circular polarization parameter). For most of our results, we will mostly deal with Stokes parameter $I$ and the degree of polarization $P$, defined as

$$
P=\frac{\sqrt{Q_{\text {planet }}^{2}+U_{\text {planet }}^{2}}}{I_{\text {star }}} .
$$

Starlight is assumed to be unpolarized, so $Q_{\text {star }}$ and $U_{\text {star }}$ are zero. Thus, polarization and reflected flux from the planet are always expressed as fractions of direct unscattered starlight in units of parts per million (ppm) or parts per billion (ppb). The Cox-Munk model uses the liquid's refractive index and the surface wind speed (as a measure of the surface roughness) as inputs to calculate the reflectance function. In the example of a glint spot as simulated by the model, (shown in Figure 1), the glint spot is strongest when the viewing zenith angle (VZA) is equal to the solar zenith angle (SZA) at an azimuthal angle (AZM) of 0 . Increasing the wind speed parameter in the CoxMunk model causes the water surface to become rougher, and smears out the glint spot to a wider range of angles. For any realistic planet, the ocean surface roughness and winds vary significantly, and using a single wind speed is a simplification. This can be thought of as the wind speed in the vicinity of the specular point on the surface that glint reflectance will be most sensitive to.

For simulating the dry land surfaces in our models, we use a Lambertian surface. Land surfaces on Earth have a wide variety of bidirectional reflectance distribution functions (BRDFs) depending on composition, minerology, surface roughness, vegetation and so forth (see e.g., Bicheron \& Leroy 2000; Bacour \& Bréon 2005). Even for surfaces made of one component, such as snow, there is still significant variability based on viewing and lighting angles, wavelength, size, and shape of snow grains (Dumont et al. 2010). While we expect some of the surface to be covered in snow based on the expected equilibrium temperatures for some of the TRAPPIST-

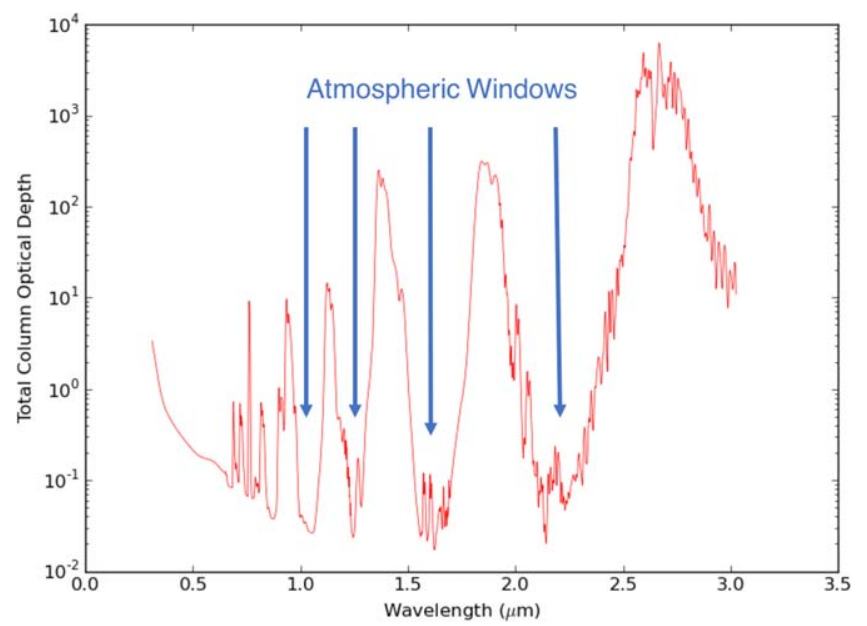

Figure 2. A typical total column optical depth spectrum of the Earth's atmosphere between the wavelengths 0.3 and $3 \mu \mathrm{m}$ with Rayleigh scattering, gas, and aerosol extinction but no clouds. Such window regions offer opportunities for observing scattered light from the planetary surface in otherwise opaque atmospheres. Note that an atmosphere can be optically thin in a given wavelength range ("atmospheric window" regions) even with a large column air mass. The aerosol optical depth here is about 0.01 , higher aerosol optical depths will make the window regions more opaque. This profile was generated by the WRF-Chem chemistry and transport model for a location over the continental US (Grell et al. 2005; Fast et al. 2006; Peckham et al. 2011).

1 planets, we are still very far from being able to observationally constrain such details as the coverage and properties of snow on the surface. Therefore, in line with our lack of information, we use a very simple surface model-an isotropic (Lambertian) surface with an albedo of 0.2 (typical land surfaces on the Earth have albedos between 0.05 and 0.4 , we simply chose a middling value). Lambertian surfaces are commonly used as first order approximations for modern high resolutions retrievals of Earth surface albedos from remote sensing instruments such as MODIS (Wang et al. 2010).

\section{Results}

\subsection{Atmospheric Effects}

Atmospheric absorption and scattering are complex effects, with strong dependencies on wavelength, observing geometry and atmospheric composition. Since we are interested in glint signals from the surface, it makes sense to choose wavelengths in which the atmosphere is most transparent. On Earth, for instance, there are several such atmospheric windows in the near-infrared regions, where the optical depth is of order $10^{-2}$ (Figure 2). The window locations may be different for TRAPPIST-1 planets, depending on the makeup of their atmospheres. Ascertaining the chemical compositions of atmospheres on these planets is outside the scope of this paper.

Since we do not know where the window regions are, it is likely that glint observations will contain some amount of atmospheric absorption. In such cases, it is important to know how thick the atmosphere of a planet can be before the glint signal is completely obscured. To estimate this, we consider the effects of a Rayleigh scattering atmosphere on top of a glinting ocean, with different optical thicknesses (Figure 3). Both scattering and absorption contribute to the atmospheric optical depth. While atmospheric scattering can change both the degree of polarization and the phase angle of peak polarization, atmospheric absorption will mostly attenuate the overall signal. 

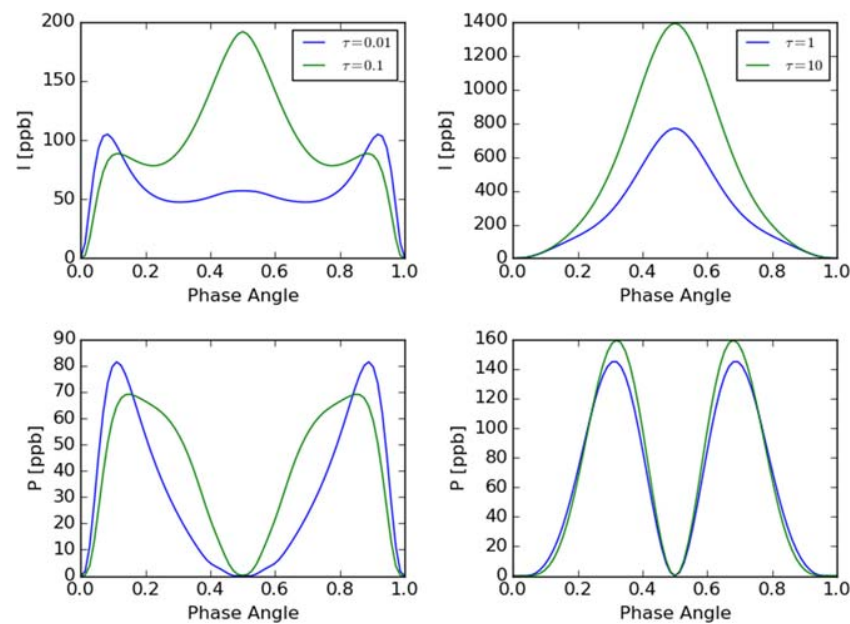

Figure 3. Reflected starlight intensity and degree of polarization phase curves for TRAPPIST-1e in the thin (left column) and thick atmospheric (right column) limits over an ocean like glinting surface. The legend indicates the optical depth of the atmosphere. The phase angle used here is an atypical convention chosen for consistency with exoplanetary polarization literature (e.g., Wiktorowicz et al. 2015). Under this convention, phase angles of 0 and 1 correspond to mid transit (only nightside is visible) and a phase of 0.5 corresponds to opposition (full dayside is visible). Intensity and polarization are expressed as fractions of direct, unscattered starlight in units of parts per billion [ppb]. Wind speed for the Cox-Munk function is always $10 \mathrm{~m} \mathrm{~s}^{-1}$ in all following figures, taking a typical value for wind speeds over oceans on Earth as reference (Sampe \& Xie 2007).

Since we are interested in glint signals from the surface, it makes sense to choose wavelengths where the atmosphere is most transparent. Other than the straightforward interpretation of optical depth being a indicator of atmospheric mass or thickness, this can also be thought of as the difference between observing at various wavelengths from 1.5 and $2 \mu \mathrm{m}$ in an Earth-like atmosphere (Figure 2), where the optical depth changes by a factor of 10000 . In the thin atmosphere limit, the glint signal is clearly visible at crescent phases (phase angles 0.1 and 0.9 ), whereas the glint signal is almost entirely absent in the thick atmospheres. The high degree of polarization in the thick atmosphere cases comes from Rayleigh scattering in the atmosphere. The thick atmosphere planets are also much brighter than those with thin atmospheres, since the atmospheres have no absorption (single scattering albedo of 0.9999) and the surface is relatively dark at angles away from the glint spot.

Clouds and hazes introduce their own features into both the intensity and polarization curves. The effects of cloud particle size and global cloud fraction have been discussed in detail in other works (e.g., Zugger et al. 2010, 2011; Karalidi et al. 2012). For our purposes, we examine one representative case of water clouds with a size of $2 \mu \mathrm{m}$ and a size variance of $0.1 \mu \mathrm{m}$, which are similar to cloud particle type A in Karalidi et al. (2012). Phase curves for TRAPPIST-1e with a planet wide deck of clouds of different optical depth shown in Figure 4. Since the TRAPPIST-1 planets are located very close-in, they are expected to be tidally locked. They will have clouds centered around the sub-stellar point, and to a first approximation, they are uniform over the dayside hemisphere (Yang et al. 2013; Yang \& Abbot 2014). Since we are interested in qualitatively examining the differences between clear and cloudy atmospheres, we study one case where the atmospheric scattering is completely dominated by clouds. There is no scattering by gas in the cloud scenarios, only
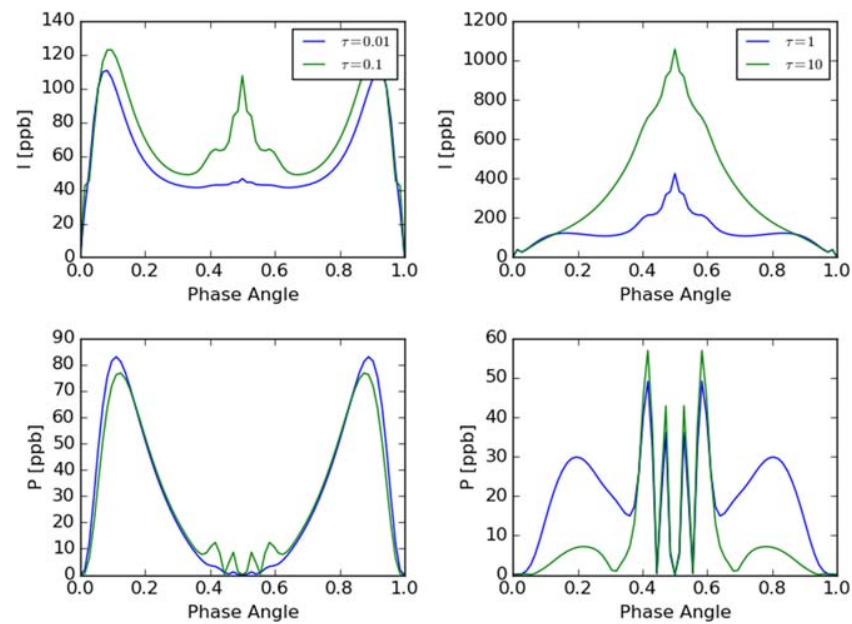

Figure 4. Same as Figure 3, but with scattering by cloud particles of size $2 \mu \mathrm{m}$. Note the appearance of rainbows around phase 0.4 and 0.6 in the thick cloud limit.

scattering by cloud particles, such that the atmosphere always has one component only. The reason for a single component atmosphere is to keep the number of free parameters in each model simulation to a minimum. This is also the intent behind using a single wind speed parameter, a single surface albedo, a single size for the cloud particles, and a single atmospheric optical depth for simulations involving all seven planets later on in the paper. For studies involving Earth, the data is of sufficient quality and volume that models can be complex, with many parameters to retrieve all the information present in the observations. For Earth-like exoplanets, from which scattered light is barely detectable, there is not enough information to justify models with a large number of free parameters. Therefore, we try to keep the models in this study as simple as possible. Thick clouds increase planet brightness significantly at opposition as compared to thin clouds, but generally have a lower degree of polarization.

\subsection{Signatures of Different Ocean Configurations}

For further modeling in this section, we will use an atmospheric optical depth of 0.1 , a surface Lambertian albedo of 0.2 (for dry surfaces), a weakly absorbing Rayleigh scattering atmosphere (single scattering albedo of 0.9999), and a wind speed of $10 \mathrm{~m} \mathrm{~s}^{-1}$ for the Cox-Munk model. A Rayleigh scattering, Earth-like atmosphere with a pressure of 1 bar can have optical depths as low as $0.01-0.001$ in window regions in the near-infrared (Figure 2). However, we will use optical depth 0.1 , because as seen in Figures 3 and 4 in the previous section, this optical depth provides a fair mixture of features from both the atmosphere and ocean glint. The previous section only dealt with a uniform, global ocean. However, this is only one possible configuration. If the planet is tidally locked and near the outside edge of the continuously habitable zone, we may have an open water ocean around the sub-stellar point. It should be noted that factors such as the total insulation, thermal mass of the atmosphere, presence of greenhouse gases, and the orbital period can affect the ocean configuration. We model this case by setting an open ocean within $30^{\circ}$ of the sub-stellar point and dry surfaces elsewhere (eyeball ocean). With a smaller fraction of ocean surface, the difference in the intensity curves is harder to detect. Furthermore, in the case where the planet is tidally locked 

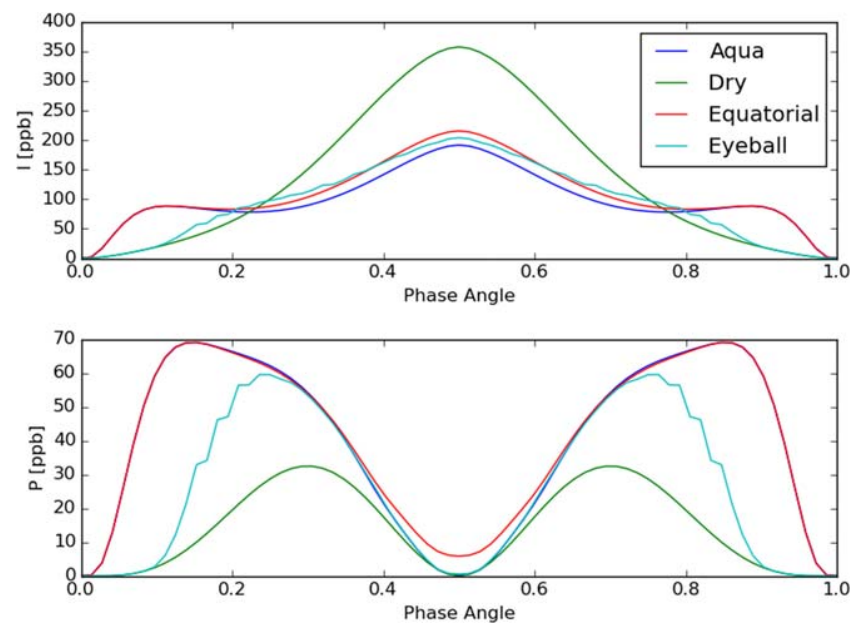

Figure 5. Phase curves for fully ocean-covered (aqua), completely dry planet and two intermediate cases. The equatorial ocean and global ocean cases have near identical polarization curves since the glint signal comes primarily from near the equator. The intensity curves for the non-dry cases are nearly identical, and different from the dry case, since the dry surfaces are brighter than the ocean at non-glint angles and contribute significantly to disk brightness.

with a short period or not tidally locked but still quite cold, we could expect to have a planet-encircling ocean around the equator. For the case of an equatorial ocean, the polarization is intermediate to the two cases discussed above. We model this case by having an ocean within $30^{\circ}$ latitude of the equator. Models for these cases (a global ocean, a wholly dry planet, eyeball ocean, and equatorial ocean) are shown in Figure 5. Planets with oceans are in general darker than the dry planet; however, the eyeball ocean curve coincides with the dry planet curve after quadrature phases $(<0.25$ or $>0.75)$ when the ocean begins to go out of view. The eyeball ocean curve shows small jumps, which are a result of spatial integration of grid boxes over the illuminated disk (64 boxes) and temporal discretization (72 phases of the planet per orbit). The other curves are smooth because their scattering properties do not vary over the disk with phase, while for the eyeball ocean, they vary as the ocean comes and goes out of view of the observer. Note that the equatorial ocean planet also a small polarization value even at a phase angle of 0.5, when all other curves fall to zero. For the net polarization over the disk to be zero, the disk must be symmetric to cancel out the positive and negative lobes of Stokes $Q$ and $U$ parameters over the surface. $Q$ and $U$ have a quadrupole structure on a uniform disk (Schmid et al. 2006). For $Q$, the maximum values lie at the intersections of the limb with the equator and the prime meridian. For $U$, they are rotated clockwise by $45^{\circ}$ to $Q$. For a homogeneously cloudy or Rayleigh scattering planet, or a planet with an eyeball ocean, these symmetries are preserved at a phase angle 0.5 leading to a net polarization of zero. For the equatorial ocean planet, symmetry is preserved for $U$. But for $Q$, the negative lobe contribution at the equator comes almost entirely from the ocean surface, while the positive lobe at the poles comes from dry land. Due to this asymmetry, a small residual net polarization remains at phase 0.5 .

\subsection{Phase Curves for the TRAPPIST-1 System}

The TRAPPIST-1 system consists of seven planets packed into orbits closer than 0.07 au around their star. Observations of reflected light curves will likely not be able to spatially separate
Table 1

Scaling Factors for Brightness to Convert Above Curves to Other Planets in the System Based on Relative Size and Orbital Distance

\begin{tabular}{lccc}
\hline \hline Planet & Radius $\left(R_{p}\right)$ & Orbit $(\mathrm{au})$ & Scaling Factor \\
\hline TRAPPIST-1b & 1.09 & 0.011 & 9.00 \\
TRAPPIST-1c & 1.06 & 0.015 & 4.54 \\
TRAPPIST-1d & 0.77 & 0.021 & 1.22 \\
TRAPPIST-1e & 0.92 & 0.028 & 1.0 \\
TRAPPIST-1f & 1.04 & 0.037 & 0.75 \\
TRAPPIST-1g & 1.13 & 0.045 & 0.59 \\
TRAPPIST-1h & 0.72 & 0.060 & 0.13 \\
\hline
\end{tabular}

Note. Values taken from Wang et al. (2017).

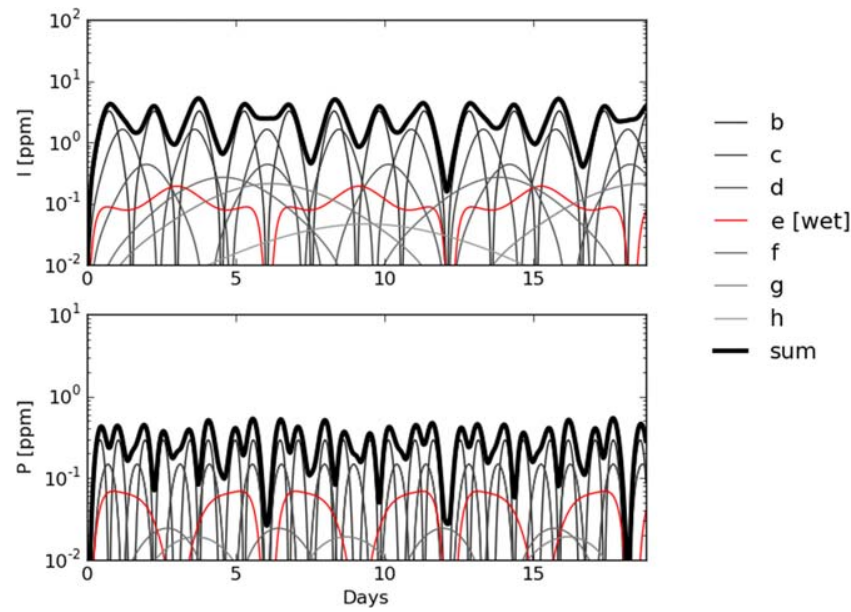

Figure 6. Reflected light intensity and polarization expressed as a fraction of direct starlight for the TRAPPIST-1 system for one period of the outermost planet ( $\sim 19$ days). Planet e is modeled as an aqua planet, while the other planets are completely dry. The wet planet signal contributes a much higher fraction of the sum total in polarization $(\sim 20 \%)$ than in intensity $(\sim 1 \%)$.

light coming from different planets. So any data collected will measure the sum of the reflected light coming from all the planets. We investigate the prospects of retrieving a glint signal from such a sum. To convert the brightness and polarization phase curves from the above calculations for TRAPPIST-1e to other planets in the system, they will need to be scaled by a factor of $R_{p}^{2} / a^{2}$, where $R_{p}$ is the radius of the planet and $a$ is the orbital distance from the star. We only model starlight scattering by the planets and do not consider star-planet interactions such as transits or eclipses in these phase curves. For convenience, we provide these factors in Table 1.

We assume that all planets other than TRAPPIST-1e are dry and spatially homogeneous (no albedo or surface BRDF variations), and TRAPPIST-1e is an aqua planet. We will use an atmospheric optical depth of 0.1 (except when otherwise mentioned), a surface Lambertian albedo of 0.2 (for dry surfaces), a weakly absorbing Rayleigh scattering atmosphere (single scattering albedo of 0.9999 , except when otherwise mentioned), and a wind speed of $10 \mathrm{~m} \mathrm{~s}^{-1}$ for the Cox-Munk model. As discussed earlier, with the intention of keeping model free parameters to a minimum, all dry planets are modeled with the same atmospheric conditions and surface albedos. Spatial homogeneity on each planet is another simplifying assumption. Figure 6 shows the reflected light curves for this case. The summed intensity and polarization as shown by the thick, black curve is the observable quantity. Note that the contribution of planet e to the sum total in 


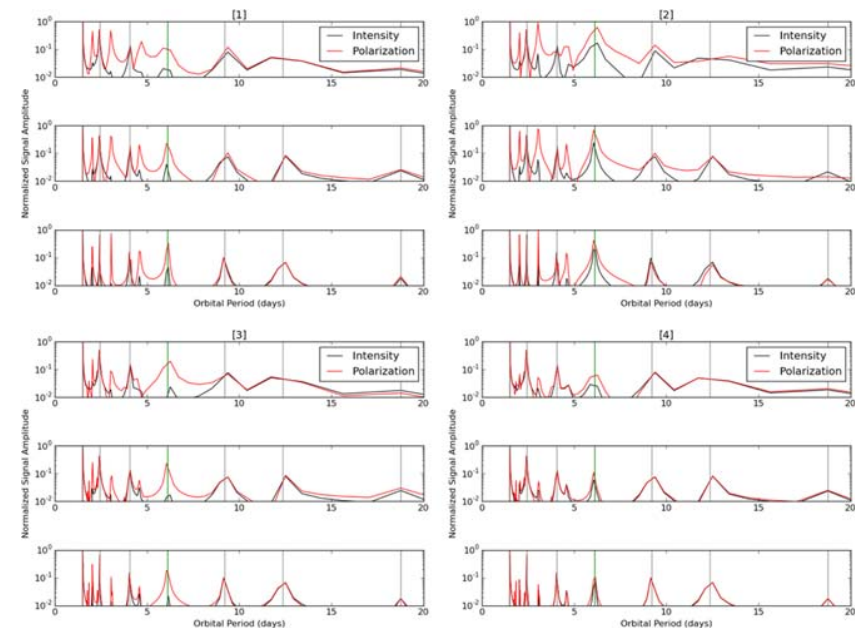

Figure 7. Fourier transforms of the combined phase curves (dark black line in Figure 6) for observing periods totaling 5, 10, and 20 periods of the outermost planet ( $~ 94,188$ and 375 days) for cases 1-4 described in the text. Vertical lines indicate the periods of the known planets, TRAPPIST-1e is in green.

intensity $(0.07 \mathrm{ppm}$ out of $5 \mathrm{ppm}$ or $\sim 1 \%)$ is about one order of magnitude smaller than its contribution to the polarization ( $0.07 \mathrm{ppm}$ out of $0.4 \mathrm{ppm}$ or $\sim 20 \%$ ). Therefore, observing polarization offers a far larger contrast in this case. Note also that having multiple planets around a star increases the degree of polarization of the star-planet system, which might be a usable characteristic for surveys.

Given this observable quantity, we want to answer the questions: how long is an observing period necessary to obtain a definitive signal in polarized light from this planet? How reliable is this detection under different conditions? To examine this, we consider the combined light curves of the TRAPPIST1 planets under a few different scenarios:

Case 1: Planet e has a global ocean, other planets are totally dry. All planets have a clear atmosphere with an optical depth of 0.1 (same as Figure 6).

Case 2: Same as Case 1, except planet e has a clear atmosphere with optical depth of 1 . Note that the polarization signal from planet $\mathrm{e}$ is now dominated by atmospheric Rayleigh scattering instead of ocean glint.

Case 3: All planets have a cloudy atmosphere with an optical depth of 0.1 . The ocean on planet $\mathrm{e}$ is visible, but slightly obscured by clouds.

Case 4: All planets have a cloudy atmosphere with an optical depth of 1, i.e., the surface is invisible on all planets.

We compute the Fourier transform of the sum total intensity and polarization curves for different observing periods for each of these cases (Figure 7). Large amplitude variations of less than 1.5 days (shortest known planetary period) are smoothed to zero. For observing periods on the order of 200 days and above, a peak corresponding to the period of planet $e$ is distinguishable by eye from the power spectrum.

For cases 1 and 3, where the glint signal is most unambiguous, the Fourier transform shows a clear signal for planet e that is about a factor of 10 stronger in polarization than in intensity. Figure 7 does not show the glint signal; it only shows that a polarization signal at the period of the ocean planet $\mathrm{e}$ is distinguishable. We know the glint signal is clear because of Figures 3 and 4 . The glint signal can only be recognized from curves as in Figure 6, which show the phase at which polarization peaks for planet e. The phase of the planet is necessary information to distinguish between glint and atmospheric scattering polarization peaks. For case 2, where the thick atmosphere gives planet e a much higher albedo, the detection is stronger than in any other case. Conversely, if the atmosphere of planet e had absorbers that lowered its albedo, both intensity and polarization will give a much weaker signal. However, polarization and intensity signals are about the same magnitude and polarization offers no advantage for observing. In case 4 , the thick cloud deck makes all the planets look uniform. The strength of the observable signal monotonically decreases with distance from the star, and polarization again offers no real advantage over intensity alone.

Note that we have not modeled transits and eclipses in our phase curves. At these points, scattered light from the planet falls to zero, which will add more noise to the power spectrum. However, even in the present simulations, the planets' polarization signal falls to zero at both mid transit and mid eclipse. We therefore do not expect a qualitative change in the polarization results due to the inclusion of these events. Additionally, TRAPPIST-1 is an active star (Roettenbacher \& Kane 2017) with a noisy stellar spectrum that contaminates planetary spectra (Zhang et al. 2018). Variations in surface type, cloud cover, cloud top height, cloud particle shape, and size will noticeably change the phase curves as seen in observation based studies of Earth as an exoplanet (Livengood et al. 2011; Robinson et al. 2011). The presence of additional polarization features from these inhomogeneities will make it significantly harder to detect glint. But, advances in understanding of the polarization signals of various cloud scenarios (Fauchez et al. 2017; Rossi \& Stam 2017) could pave the way to isolate at least some of these effects. In summary, polarization offers great advantages for glint detection under certain conditions, but variability in planetary properties and stellar noise or instrumental polarization (e.g., Wiktorowicz et al. 2015) can limit how useful the technique can be.

\subsection{Observing Possibilities}

For the case of TRAPPIST-1, the dimness of the star, the small size of the planet, and the presence of several other planets in the system make it extremely challenging to observe phase curves associated with surface reflectance without committing several thousand hours of observing time from the largest ground-based telescopes. This is in spite of the polarized glint signal offering a $10 \times$ better contrast than a simple intensity phase curve. Present-day ground and space telescopes cannot reach this high contrast at small angular separations from the star (Mawet et al. 2012; Robinson et al. 2016). For the moment, the way to indirectly infer the presence of an ocean on one of the TRAPPIST-1 planets will be the detection of water absorption in the planetary atmosphere during transit along with some measurement or estimate of surface temperature.

In the near future, there are proposals for several large spaceand ground-based telescopes with coronagraphs (and polarimeters), which make them sensitive to very low planet-star contrasts. Here we explore the parameter space of where ocean planets might exist and what instruments might be able to observe their glint signals (Figure 8). We scale our aqua-planet modeling results for TRAPPIST-1e to Earth-sized planets at different orbital distances around stars of various luminosities. Since the degree of polarization is close to $100 \%$, the glint 


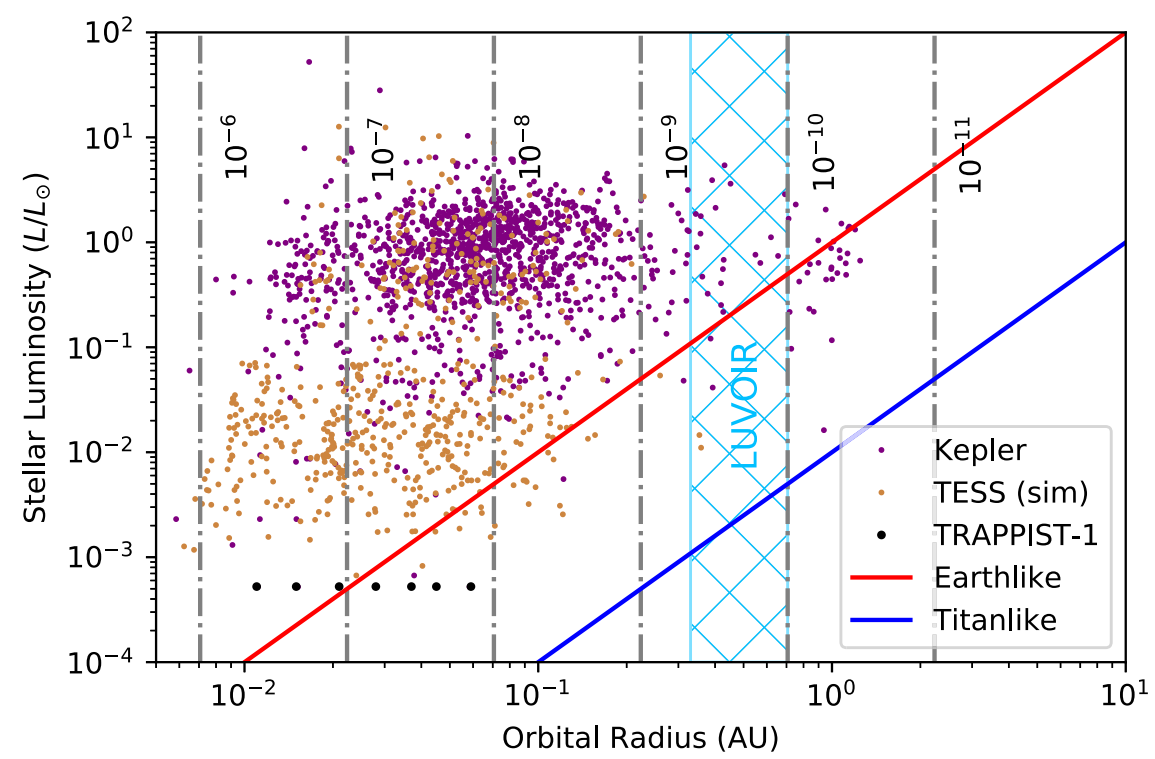

Figure 8. Modeled glint star-planet contrast signals (dot-dash gray lines) plotted against detection limits of the upcoming large telescope, LUVIOR, for systems at a distance of $10 \mathrm{pc}$. The blue and red lines indicate the distance from the star at which a planet receives an Earth-like (surface water ocean) and Titan-like (surface hydrocarbon lakes) solar flux. Present or near future observable Earth-like planets (size smaller than $2 R_{\oplus}$ ) from Kepler detections and candidate objects (downloaded from exoplanets.org (Han et al. 2014) on 2017 October 6) and simulated detections from TESS (Sullivan et al. 2015) are overplotted for a sense of the number of detections by surveys that may have an ocean, which can be followed up for glint studies. Planetary periods listed in the TESS simulated data set are converted to orbital distances by converting the listed stellar radii to stellar masses using the method of Demircan \& Kahraman (1991).

polarization and intensity contrasts with respect to the star are the same.

WFIRST-AFTA (Spergel et al. 2015) is a $2.4 \mathrm{~m}$ space telescope with a coronagraph (and possibly a polarimeter) and is expected to reach sensitivites of $0.1 \mathrm{ppb}(1 / 10000 \mathrm{ppm})$ in the visible/near-infrared. However, the coronagraph has an inner working angle (IWA) of 100 mas (milliarcseconds), which rules out the habitable zones of cool stars. The sensitivity of this instrument is about a factor of 3-4 too small to observe glint from Earth-like planets around Sun-like stars. JWST NIRCam cannot observe such signals even though it has an IWA of $\sim 10$ mas for certain configurations; the detection contrasts required are in the range of $10^{-4}$ (Beichman et al. 2010; Krist et al. 2010). The most promising space telescope concept for these observations is LUVOIR (Bolcar et al. 2015). With a proposed telescope mirror of about $18 \mathrm{~m}$, this telescope is planned to be sensitive to $10^{-10}$ contrast at 35 mas.

Among ground-based telescopes, the EPICS exoplanet imager for the $40 \mathrm{~m}$ ELT (Kasper et al. 2010) aims to achieve a contrast of $10^{-8}$ at 30 mas and $10^{-9}$ at 100 mas in the NIR, which again is about a factor of 3 smaller than the needed sensitivity. The LMIRCam on the Large Binocular Telescope (Defrere et al. 2014) can also see this range, but only reaches a contrast of $10^{-5}$ for the region of interest.

\section{Conclusions}

We model scattering of starlight by the atmosphere and surface of TRAPPIST-1e to produce a number of polarized phase curves for different distributions of the surface ocean and atmospheric cloud opacities. We find that the existence of a sizeable liquid water surface will show a strong signal in polarized light if the atmosphere is not opaque, and this measurement might offer a definitive difference between liquid water holding and dry planets. However, for the case of TRAPPIST-1, direct observation of glint polarization is impossible with current instruments. Future telescopes, such as LUVOIR may be capable of making such measurements for Earth-like planets around Sun-like stars. The presence of polarimeters on such telescopes could allow for definitive detections of oceans on Earth-like exoplanets.

We thank Peter Gao for insightful comments and Sloane Wiktorowicz for giving us an observer's perspective on the model results. We also want to thank the anonymous reviewer for their extraordinary commitment to helping us improve this paper. This research was supported in part by an NAI Virtual Planetary Laboratory grant from the University of Washington to the Jet Propulsion Laboratory (JPL) and California Institute of Technology (CIT), and in part by JPL. P.K., M.R.S., and Y. L.Y. acknowledge support from the President's and Director's Fund of CIT and JPL. We acknowledge use of the Exoplanet Orbit Database and the Exoplanet Data Explorer at exoplanets. org. We thank Jeremy Bailey, Lucyna Chudczer, and Kim Bott for helping discover a bug in our model code.

\section{ORCID iDs}

Pushkar Kopparla iㅣ https://orcid.org/0000-0002-8951-3907

Vijay Natraj (1) https://orcid.org/0000-0003-3154-9429

David Crisp iㅏ https://orcid.org/0000-0002-4573-9998

\section{References}

Alberti, T., Carbone, V., Lepreti, F., \& Vecchio, A. 2017, ApJ, 844, 7 Atlas, R., Hoffman, R. N., Ardizzone, J., et al. 2011, BAMS, 92, 157 Bacour, C., \& Bréon, F.-M. 2005, RSEnv, 98, 80

Beichman, C. A., Krist, J., Trauger, J. T., et al. 2010, PASP, 122, 162 Bicheron, P., \& Leroy, M. 2000, JGRD, 105, 26669

Bolcar, M. R., Balasubramanian, K., Clampin, M., et al. 2015, Proc. SPIE, 9602, 960209

Bourrier, V., de Wit, J., Bolmont, E., et al. 2017, ApJ, 154, 121

Cowan, N. B., Abbot, D. S., \& Voigt, A. 2012, ApJL, 752, L3

Cowan, N. B., Agol, E., Meadows, V. S., et al. 2009, ApJ, 700, 915

Cox, C., \& Munk, W. 1954, JOSA, 44, 838 
Defrere, D., Absil, O., Hinz, P., et al. 2014, Proc. SPIE, 9148, 91483X

Demircan, O., \& Kahraman, G. 1991, Ap\&SS, 181, 313

Dumont, M., Brissaud, O., Picard, G., et al. 2010, ACP, 10, 2507

Fast, J. D., Gustafson, W. I., Easter, R. C., et al. 2006, JGRD, 111

Fauchez, T., Rossi, L., \& Stam, D. M. 2017, ApJ, 842, 41

Gillon, M., Triaud, A. H., Demory, B.-O., et al. 2017, Natur, 542, 456

Grell, G. A., Peckham, S. E., Schmitz, R., et al. 2005, AtmEn, 39, 6957

Han, E., Wang, S. X., Wright, J. T., et al. 2014, PASP, 126, 827

Horak, H. G. 1950, ApJ, 112, 445

Karalidi, T., Stam, D., \& Hovenier, J. 2012, A\&A, 548, A90

Kasper, M., Beuzit, J.-L., Verinaud, C., et al. 2010, Proc. SPIE, 7735, 77352E

Kopparla, P., Natraj, V., Zhang, X., et al. 2016, ApJ, 817, 32

Krist, J. E., Balasubramanian, K., Muller, R. E., et al. 2010, Proc. SPIE, 7731, $77313 \mathrm{~J}$

Lingam, M., \& Loeb, A. 2017, PNAS, 114, 6689

Livengood, T. A., Deming, L. D., A'Hearn, M. F., et al. 2011, AsBio, 11, 907

Luger, R., Sestovic, M., Kruse, E., et al. 2017, NatAs, 1, 0129

Mawet, D., Pueyo, L., Lawson, P., et al. 2012, Proc. SPIE, 8442, 844204

Meadows, V. S., Reinhard, C. T., Arney, G. N., et al. 2017, arXiv:1705.07560

O’Malley-James, J. T., \& Kaltenegger, L. 2017, MNRAS: Letters, 469, L26

Peckham, S., Fast, J., Schmitz, R., et al. 2011, WRF/Chem Version 3.3 User's Guide, 94
Robinson, T. D., Meadows, V. S., \& Crisp, D. 2010, ApJL, 721, L67

Robinson, T. D., Meadows, V. S., Crisp, D., et al. 2011, AsBio, 11, 393

Robinson, T. D., Stapelfeldt, K. R., \& Marley, M. S. 2016, PASP, 128, 025003

Roettenbacher, R. M., \& Kane, S. R. 2017, ApJ, 851, 77

Rossi, L., \& Stam, D. 2017, A\&A, 607, A57

Sampe, T., \& Xie, S.-P. 2007, BAMS, 88, 1965

Schmid, H., Joos, F., \& Tschan, D. 2006, A\&A, 452, 657

Spergel, D., Gehrels, N., Baltay, C., et al. 2015, arXiv:1503.03757

Spurr, R. J. 2006, JQSRT, 102, 316

Stam, D. 2008, A\&A, 482, 989

Sullivan, P. W., Winn, J. N., Berta-Thompson, Z. K., et al. 2015, ApJ, 809, 77

Wang, S., Wu, D.-H., Barclay, T., \& Laughlin, G. P. 2017, arXiv: 1704.04290

Wang, Y., Lyapustin, A. I., Privette, J. L., et al. 2010, RSEnv, 114, 2791

Wiktorowicz, S. J., Nofi, L. A., Jontof-Hutter, D., et al. 2015, ApJ, 813, 48

Wolf, E. T. 2017, ApJL, 839, L1

Yang, J., \& Abbot, D. S. 2014, ApJ, 784, 155

Yang, J., Cowan, N. B., \& Abbot, D. S. 2013, ApJL, 771, L45

Zhang, Z., Zhou, Y., Rackham, B., \& Apai, D. 2018, arXiv:1802.02086

Zugger, M., Kasting, J., Williams, D., Kane, T., \& Philbrick, C. 2011, ApJ, 739,12

Zugger, M. E., Kasting, J. F., Williams, D. M., Kane, T. J., \& Philbrick, C. R. 2010, ApJ, 723, 1168 